Proc. 13th International School on Theoretical Physics: Symmetry and Structural Properties of Condensed Matter

\title{
Boltzmann Approach to the Problem of Transient Currents in Electronic Devices
}

\author{
K. Kulinowski, M. WoŁoszyn And B.J. SpisAK* \\ AGH University of Science and Technology, Faculty of Physics and Applied Computer Science, \\ al. Mickiewicza 30, 30-059 Kraków, Poland
}

\begin{abstract}
The kinetic method based on the time-dependent Boltzmann transport equation is applied to the problem of transient phenomena in open systems. For this purpose the transport equation is numerically solved and the zeroth and first moments of the distribution function are determined as functions of time. The developed procedure allows one to find the transient current flowing through the system and to determine the equilibrium limit of these transport processes.
\end{abstract}

DOI: 10.12693/APhysPolA.135.1271

PACS/topics: Boltzmann equation, relaxation time, scattering, transient phenomena, electronic transport

\section{Introduction}

Reliable operation of electronic devices and the possibility of controlling their properties is one of the crucial factors for their application. Therefore it requires not only experimental and theoretical knowledge of transport phenomena but also numerical simulations which are a powerful research method used to investigate physical phenomena in such systems. An interesting class of processes which occur in electronic systems under the influence of a perturbation resulting from the applied electric field are transient phenomena. They represent a response of the electronic system arising from switching on the bias voltage. In such situations electrons are precipitated from equilibrium which leads to the creation of a current pulse decaying before the device reaches a stable state.

In this report we present results showing the transient response of an electronic device to a perturbation due to the electric field. For this purpose we determine the electronic distribution function by solving the timedependent Boltzmann transport equation with appropriate initial and boundary conditions. We then calculate the electronic current as the response of the considered system to the bias voltage applied between contacts before reaching the steady state.

\section{Model, theory and methods of calculations}

We consider a model of a two-terminal electronic device which consists of two contacts and an active region made of amorphous GaAs compounds. A schematic of the device is shown in Fig. 1. We assume that the contacts are characterized by the equilibrium distribution functions of electrons with their own electrochemical potential and temperature, while the active region of the

*corresponding author; e-mail: bjs@agh.edu.pl

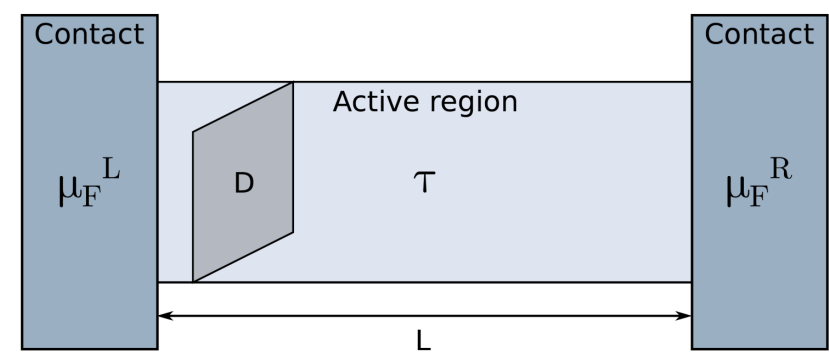

Fig. 1. Schematic view of the two-terminal electronic device. Active region of the system is sandwiched between the contacts.

system is structurally defected and we treat it as a disordered system where the scattering processes are described by the relaxation time [1].

Transport properties of the considered system can be derived from the non-equilibrium distribution function $f(x, p, t)$ for the carriers which satisfies the timedependent Boltzmann transport equation in the form

$$
\begin{gathered}
\frac{\partial}{\partial t} f(x, p, t)+v(p) \frac{\partial}{\partial x} f(x, p, t)= \\
-\frac{1}{\tau}\left[f(x, p, t)-f^{0}(p)\right],
\end{gathered}
$$

where $\tau$ is the relaxation time which characterizes the elastic scattering processes of carriers, $v(p)=p / m^{*}$ is the velocity of conduction electrons which stems from the quadratic dispersion relation $E(p)=p^{2} / 2 m^{*}$ with the effective mass $m^{*}$, and $f^{0}(p)$ is the equilibrium distribution function. The kinetic Eq. (1) is supplemented by the inflow boundary condition in the form [2]:

$$
\begin{aligned}
& \left.f(x=0, p)\right|_{p>0}=f^{L}(E(p)), \\
& \left.f(x=L, p)\right|_{p<0}=f^{R}(E(p)),
\end{aligned}
$$

where $f^{L(R)}(E(p))$ are the supply functions for the left $(L)$ and right $(R)$ contacts [3]: 


$$
\begin{aligned}
& f^{L(R)}(E(p))=\frac{m^{*} k_{\mathrm{B}} T}{\pi \hbar^{2}} \\
& \quad \times \ln \left(\exp \left(-\frac{E(p)-\mu_{\mathrm{F}}^{L(R)}}{k_{\mathrm{B}} T}\right)+1\right),
\end{aligned}
$$

where $k_{\mathrm{B}}$ is the Boltzmann constant, $T$ is the temperature, and $\mu_{\mathrm{F}}^{L(R)}$ is the electrochemical potential in the left (right) contact. The difference between the electrochemical potentials of the left and right reservoirs corresponds to the applied bias voltage between them, i.e., $e V_{B}=\mu_{\mathrm{F}}^{L}-\mu_{\mathrm{F}}^{R}$. In turn, the initial condition for Eq. (1) is given by a Gaussian function centred in the phase space around the point $\left(x_{0}, p_{0}\right)$ at time $t=0$ and the initial half-widths $\delta_{x}$ and $\delta_{p}$. Therefore, the form of the initial condition is given by the formula

$$
f(x, p, 0)=A \exp \left(-\frac{1}{2 \delta_{p}^{2}}\left(p-p_{0}\right)^{2}-\frac{1}{2 \delta_{x}^{2}}\left(x-x_{0}\right)^{2}\right),
$$

where $A$ is the amplitude of the Gaussian determined according to the normalization condition in the form

$$
\int \mathrm{d} x \mathrm{~d} p f(x, p)=\frac{N}{D},
$$

where $D$ is the cross section, and $N$ represents the number of electrons injected to the active region of the electronic device from the left contact. All numerical calculations are performed on a computational grid $N_{x} \times N_{k}$ with $N_{x}=100$ mesh points for the position $x$ and $N_{k}=100$ for the wave vector $k$. This corresponds to the following discretization of the phase space:

$$
\begin{cases}x_{i}=i \Delta_{x}, & i=0,1, \ldots, N_{x}-1, \\ k_{j}=\Delta_{k}\left(j-\frac{1}{2}\left(N_{k}-1\right)\right), & j=0,1, \ldots, N_{k}-1,\end{cases}
$$

where $\Delta_{x}=L / N_{x}$ and $\Delta_{k}=2 k_{\max } / N_{k}$.

The distribution function $f(x, k, t)$ in the discrete form is denoted by $f_{i, j, l}$. In turn, the discrete version of Eq. (1), developed for the numerical scheme based on the Crank-Nicholson method, can be written in the form

$$
\frac{1}{\Delta_{t}}\left(f_{i, j, l}-f_{i, j, l-1}\right)=\frac{1}{2}(\hat{\boldsymbol{K}}+\hat{\boldsymbol{S}})\left[f_{i, j, l}+f_{i, j, l-1}\right],
$$

where $\hat{\boldsymbol{K}}$ is the diffusion term approximated using the second order finite difference method,

$$
\begin{aligned}
& \hat{\boldsymbol{K}}\left[f_{i, j, l}\right]=\frac{\hbar k_{j}}{m^{*}} \frac{\mathrm{d} f_{i, j, l}}{\mathrm{~d} x} \\
& \approx \frac{\hbar k_{j}}{m^{*} \Delta_{x}} \begin{cases}f_{i, j, l}-f_{i-1, j, l}, & k>0, \\
-f_{i, j, l}+f_{i+1, j, l}, & k<0,\end{cases}
\end{aligned}
$$

while the scattering term $\hat{\boldsymbol{S}}$ is given by

$$
\hat{\boldsymbol{S}}\left[f_{i, j, l}\right]=\frac{f_{i, j, l}-f_{j}^{0}}{\tau} .
$$

The calculations are performed with respect to the Courant-Friedrichs-Lewy condition for convergence, i.e., $\Delta_{t} v_{\max } / \Delta_{x} \leq C_{\max }$, where $C_{\max }$ is the Courant number which for algorithmic stability cannot be larger than one and therefore $\Delta_{t} \leq \Delta_{x} m^{*} / p_{\max }$.
We numerically solve the Boltzmann transport Eq. (1) with the conditions given by Eqs. (2) and (4) for a fixed bias voltage and relaxation time using the discrete form given by Eq. (7). As a result, the time-dependent distribution function $f\left(x, p, t ; V_{B}, \tau\right)$ is determined and then the current density is calculated according to the formula

$$
j\left(x, t ; V_{B}, \tau\right)=\frac{e}{2 \pi} \int \mathrm{d} p v(p) f\left(x, p, t ; V_{B}, \tau\right),
$$

which allows us to calculate the time-dependent current for the fixed bias voltage and relaxation time,

$$
I\left(t ; V_{B}, \tau\right)=\frac{1}{L} \int \mathrm{d} x j\left(x, t ; V_{B}, \tau\right),
$$

where $L$ is the length of the active region of the device. It is assumed that the stationary electronic current $I_{S}\left(V_{B}, \tau\right)$ is reached after time $t_{S}$, if the following condition [4] is fulfilled,

$$
\left.\frac{\mathrm{d} I\left(t ; V_{B}, \tau\right)}{\mathrm{d} t}\right|_{t \geq t_{S}}=0 .
$$

In the presented calculations the condition (12) is checked using the third order backward finite difference method with the machine-precision accuracy.

\section{Results and discussion}

The model of the electronic device presented in Sect. 2 is used to find the transient current through the system as a response to the bias voltage applied between the contacts. The numerical calculations are performed for a system having length $L=200 \mathrm{~nm}$ and at a temperature $T=77 \mathrm{~K}$. Additionally, we assume that the effective mass of the carriers is $m^{*} / m_{0}=0.067$ and the relaxation time $\tau$ is equal to $10^{-12} \mathrm{~s}$. Our calculations proved that the transient response of the system manifests itself by the current pulse created after switching on the device, as it is shown in Fig. 2. This pulse decays after some time and the current achieves a stationary form, i.e., it is independent of time.

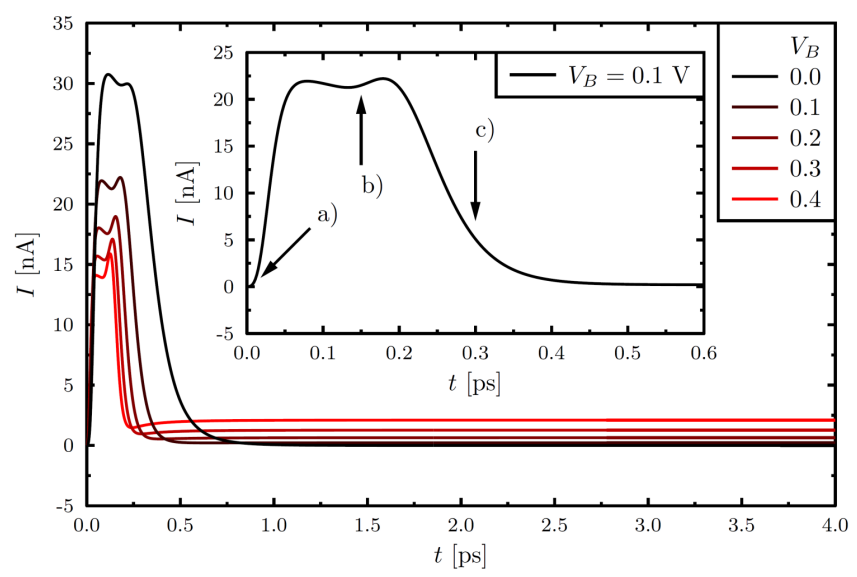

Fig. 2. The transient current $I$ as a function of time for different values of the bias voltage $V_{B}$ and for a fixed value of the relaxation time $\tau=10^{-12} \mathrm{~s}$. 

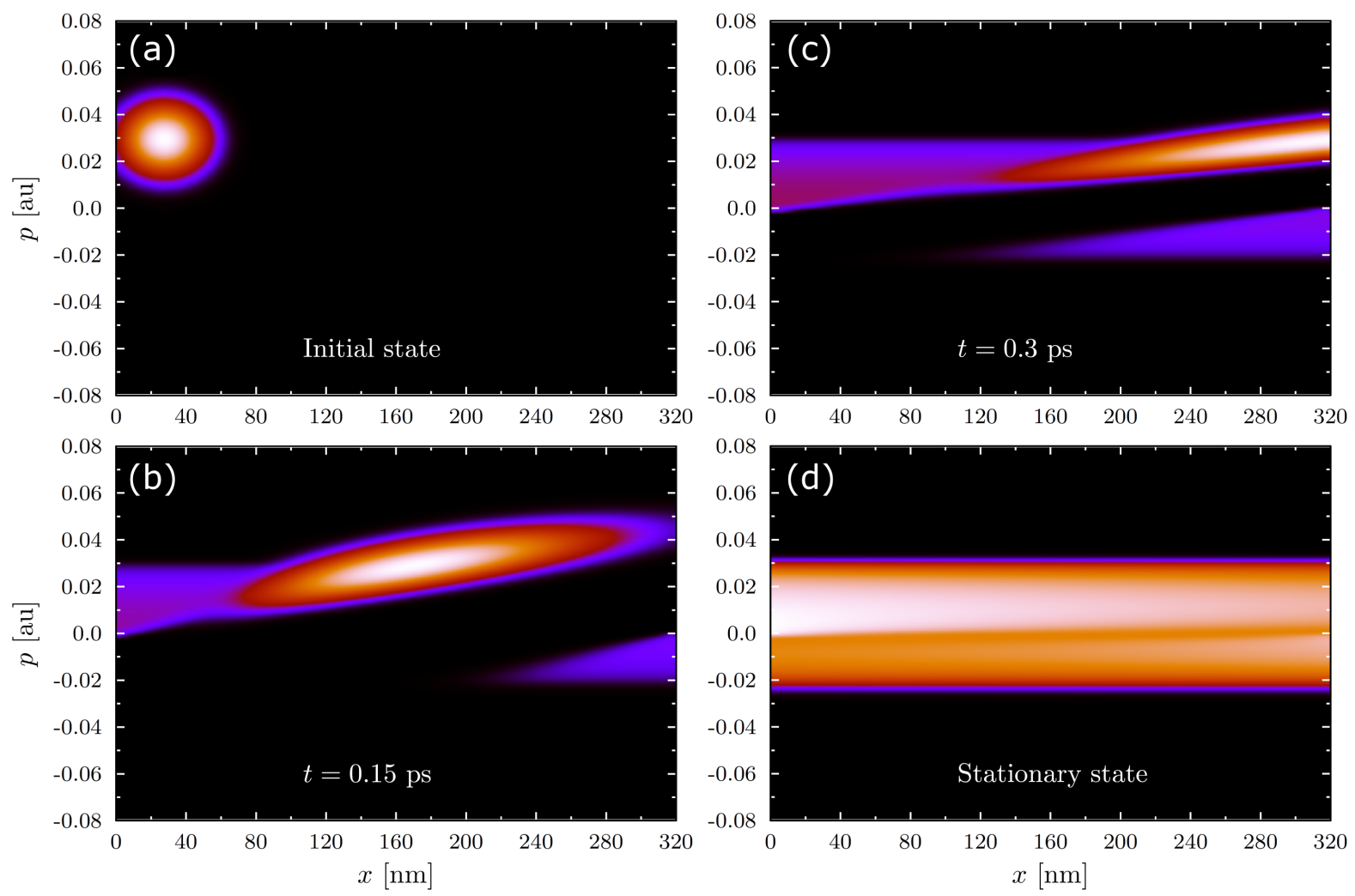

Fig. 3. The distribution function $f(x, p)$ for the bias voltage $V_{B}=0.1 \mathrm{~V}$ at (a) $t=0$, (b) $t=0.15 \mathrm{ps,} \mathrm{(c)} t=0.3 \mathrm{ps}$ and $(\mathrm{d})$ after reaching the steady state.

Figure 3 displays the phase-space snapshots of the time evolution of the non-equilibrium distribution function for the bias voltage $V_{B}=0.1 \mathrm{~V}$ at certain time instants. At $t=0$ carriers injected to the active region of the system form the Gaussian located at $x_{0}=27.56 \mathrm{~nm}$ with $\delta_{x}=9.19 \mathrm{~nm}$ and $p=0.038$ a.u. with $\delta_{p}=0.0057$ a.u. (where momentum is given in the atomic units, i.e., assuming $\hbar=e=m_{0}=1$ ), as it is presented in Fig. 3a. Figure $3 \mathrm{~b}, \mathrm{c}$ shows the motion of the state from the left to the right contact under influence of the bias voltage in the transient regime of the current characteristics.

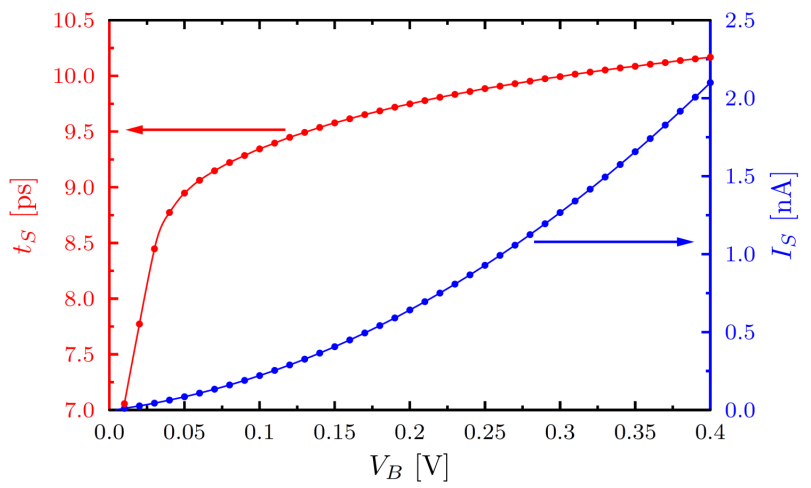

Fig. 4. The steady-state current $I_{s}$ and the time $t_{S}$ required to reach the steady state as functions of $V_{B}$.
In both these cases, we can observe the penetration of carriers into the active region from the contacts as it is expected. Finally, in Fig. 3d, we can observe the stationary form of the distribution function which is responsible for the stationary current.

We also determined the time $t_{c}$ after which the electronic current reaches the steady state based on the condition (12). It allows us to obtain the current-voltage characteristics $I_{S}-V_{b}$ displayed in Fig. 4, which also shows the time $t_{S}$ required to reach the steady state.

\section{Conclusions}

In this report, we presented an algorithm for investigating transient phenomena in electronic devices within the kinetic theory based on the Boltzmann transport equation. We have applied the algorithm to investigate the transient response of a two-terminal electronic device to a bias voltage applied between contacts. We have shown that a current pulse is formed after switching on the device, and determined the characteristic time which can be treated as a measure of the device "inertia" to the electric field before the current achieves the steady state. Simultaneously we have obtained the currentvoltage characteristics for the considered system, which has been possible owing to the precise determination of the steady state current. 


\section{Acknowledgments}

K.K. has been partly supported by the EU Project POWR.03.02.00-00-I004/16 and by the AGH UST dean grant No. 05.11220.717/45 for Ph.D. students and young researchers within subsidy of the Ministry of Science and Higher Education. B.J.S. and M.W. have been partially supported by the Faculty of Physics and Applied Computer Science AGH UST statutory tasks within subsidy of Ministry of Science and Higher Education.

\section{References}

[1] K. Kulinowski, M. Wołoszyn, B.J. Spisak, Acta Phys. Pol. A 134, 923 (2018).

[2] W.R. Frensley, Rev. Mod. Phys. 62, 745 (1990).

[3] D.K. Ferry, S.M. Goodnick, J. Bird, Transport in Nanostructures, Cambridge University Press, Cambridge 2009.

[4] D. Szydłowski, M. Wołoszyn, B.J. Spisak, Semicond. Sci. Technol. 28, 105022 (2013). 